\title{
Coexistent tophaceous gout and ankylosing spondylitis
}

\author{
I. PORTEK, B. P. WORDSWORTH, AND A. G. MOWAT \\ From the Rheumatology Unit, Nuffield Orthopaedic Centre, Oxford
}

\begin{abstract}
There has recently been considerable interest in the rare coexistence of gout and other inflammatory arthridites. ${ }^{1-3}$ We report the first case of tophaceous gout and anklyosing spondylitis.
\end{abstract}

\section{CASE REPORT}

A 62 year old man was admitted in June 1982 with persistently active polyarticular tophaceous gout and pronounced pedal oedema due to congestive cardiac failure.

The first attack of right podagra in 1969 was followed by progression to a symmetrical chronic arthritis, initially affecting only the lower limbs, and he was treated with various non-steroidal agents as well as colchicine. In 1976 arthritis developed in several predominantly distal interphalangeal joints of both hands associated with tophi. Allopurinol was added to his treatment regimen, resulting in exfoliative dermatitis. Upper limb involvement progressed to wrists, elbows, and shoulders, sparing all metacarpophalangeal joints. Further symptomatic deterioration followed a myocardial infarction in 1978 with the introduction of diuretic treatment. There was persistent hyperuricaemia and enlargement of tophi. He was placed on high doses of probenicid, which was changed to sulphinpyrazone after a poor response.
In 1965 he had suffered from low back pain radiating to his thighs. Although symptoms were attributed to mechanical back pain and a corset was prescribed, review of the radiographs showed bilateral sacroiliitis. Pain improved over the next 18 months and axial symptoms had only recurred in the past few years, within neck stiffness and a stooped posture.

On examination he was overweight with a pronounced dorsal kyphosis. Movements of the cervical and lumbar spine were considerably limited. Chest expansion was $1 \mathrm{~cm}$. Most joints except metacarpophalangeal and hip joints showed chronic inflammatory arthritis. The interphalangeal joint of his right thumb was acutely inflamed. Tophi were present on several digits and on the pinna of the ears. There was cardiomegaly but no evidence of aortic incompetence or heart failure. Pitting oedema to his knees was associated with eczematous skin changes.

$X$-ray films of the hand showed tophaceous gouty arthritis and the sacroiliac joints showed typical changes of progressive ankylosing spondylitis. Before admission the serum uric acid was persistently raised-for example, 0.778 $\mathrm{mmol} / 1$ —at admission it was 0.484 $\mathrm{mmol} / \mathrm{l} .24$ hour urate excretion was $4 \cdot 8 \mathrm{mmol}(3 \cdot 5-4 \cdot 2 \mathrm{mmol})$ without purine restriction and taking sulphinpyrazone $200 \mathrm{mg}$ four times daily. Blood urea was raised $(9.4 \mathrm{mmol} / \mathrm{l}$ $(2 \cdot 5-6 \cdot 7 \mathrm{mmol} / \mathrm{l}))$ and creatinine clearance decreased $(45 \mathrm{ml} / \mathrm{min}(105$ $\mathrm{ml} / \mathrm{min})$ ). He was HLA-B27 positive.

The absence of previous reports of the coexistence of these two diseases is surprising considering their high incidence in men, their relative frequency in the population and the fact that neither joint involvement nor serological examination would result in the diagnosis of one condition in preference to the other.

\section{References}

1 Atdjian M, Fernandez-Madrid F. Coexistence of chronic tophaceous gout and rheumatoid arthritis. $J$ Rheumatol 1981; 8: 989-92.

2 Helliwell M, Crisp A J, Grahame R. Coexistent tophaceous gout and systemic lupus erythematosus. Rheumatol Rehabil 1982; 21: 161-3.

3 Wall B A, Agudelo C A, Weinblatt M E, Tume R A. Acute gout and systemic lupus erythematosus: report of 2 cases and literature review.J Rheumatol 1982; 9: 305-7.

\section{Normal response to monosodium urate (MSU) crystals by patients with rheumatoid arthritis}

\author{
MICHAEL DOHERTY, JUNE HORNBY, AND PAULA. DIEPPE
}

From the University Department of Medicine, Bristol Royal Infirmary, Bristol BS2 $8 H W$

It has been suggested that the negative correlation between rheumatoid arthritis and gout might result from inability of patients with rheumatoid arthritis to respond to monosodium urate (MSU) crystals, perhaps due to crystal coating by rheumatoid factors with subsequent exclusion from interaction with cellular and non-cellular mediators of inflammation. ${ }^{1}$ To test the validity of this hypothesis we investigated the 
ability of patients with rheumatoid arthritis to respond to preformed crystals using both in vivo and in vitro techniques.

Needle-shaped MSU crystals 2-10 $\mu \mathrm{m}$ long were prepared by modification of the method of Seegmiller et al. ${ }^{2}$; chemical purity was checked by infrared spectrophotometry. Crystals were suspended in sterile saline (skin testing and C3 activation) or Eagle's medium (polymorphonuclear (PMN) interaction) and briefly sonicated before use to reduce aggregation.

(1) Intradermal skin testing. The diameter of erythema produced by intradermal injection of MSU crystals ( $5 \mathrm{mg}$ in $0.2 \mathrm{ml}$ suspension) was measured by a single observer as previously described. ${ }^{3}$ Fifteen patients with seropositive rhe umatoid arthritis (mean age $49 \cdot 1$ years, mean DAT 1:640), 15 patients with osteoarthritis (mean age 63.8 years), and 12 normal controls (mean age 26.2 years) showed no significant difference in mean forearm erythema 24 and 48 hours after injection (patients with rheumatoid arthritis: $29 \cdot 8 \pm 6 \cdot 2 \mathrm{~mm}$ after $24,27 \cdot 8 \pm 7 \cdot 3$ after 48 ; patients with osteoarthritis: $32.4 \pm 4.4$ and
$14 \cdot 7 \pm 8 \cdot 1 ;$ and controls $12 \cdot 2 \pm 6$ $10 \cdot 8 \pm 5 \cdot 1)$.

(2) Polymorph phagocytosis and enzyme release. PMNs from patients with rheumatoid arthritis $(n=10)$ and controls $(n=10)$ exposed to MSU crystals (1\%) for 1 hour showed no significant difference in cell attachment/phagocytosis, enzyme release or viability.

(3) Complement activation. Serum C 3 activation produced by 30 minutes' incubation with MSU crystals (1\%) was quantified by densitometry after electrophoretic separation and immunofixation as previously described. ${ }^{4}$ No difference was observed between 10 patients with rheumatoid arthritis (mean DAT $1: 640$ ) and 10 normal sera in the $\%$ of C3 split in excess of saline control $(37.8 \pm 4.9 \%$ and $36 \cdot 7 \pm 3.2 \%$ respectively). C3 activation via the alternative pathway-demonstrated by incubation with EGTA ( $8 \mathrm{mmol})$ and $\operatorname{MgCl}_{2}(0.3 \mathrm{mmol})$-was also similar $(21 \cdot 1 \pm 4 \cdot 2 \%$ and $18.5 \pm$ $3 \cdot 1 \%$ respectively).

These results show that patients with seropositive rheumatoid arthritis mount normal in vitro and in vivo inflammatory responses to MSU crystals. We therefore discount previous suggestions that the negative correlation between rheumatoid arthritis and gout results from a difference in host response. A more plausible explanation is that the connective tissues of patients with rhe umatoid arthritis are not conducive to MSU crystal formation.

We would like to acknowledge the financial support of the Arthritis and Rheumatism Council.

\section{References}

1 Wallace D J, Klinenberg J R, Morhairn D, Berlanstein B, Biren P C, Callis G. Coexistent gout and rheumatoid arthritis: case report and literature review. Arthritis Rheum 1979; 22: 81-6.

2 Seegmiller J E, Howell D R, Malawista $S$ E. The inflammatory reaction to sodium urate. JAMA 1962; 180: 469-77.

3 Dieppe P A, Doherty M, Papadimitriou $\mathrm{G} M$. Inflammatory responses to intradermal crystals in healthy volunteers and patients with rheumatic diseases. Rheumatology International 1982; 2: 55-8.

4 Doherty M, Whicher J T, Dieppe P A. Activation of the alternative pathway of complement by monosodium urate monohydrate crystals and other inflammatory particles. Ann Rheum Dis (in press).

\title{
Ultrastructural observations of crystals in articular cartilage of aged human hip joints
}

\author{
IRENIA MARANTE, R. MACDOUGALL, A. ROSS, AND R. A. STOCKWELL \\ From the Department of Anatomy, University Medical School, Edinburgh EH8 $9 A G$
}

The occurrence of calcification in the normally uncalcified zones of human articular cartilage is well documented ${ }^{1}$ and crystal morphology at the ultrastructural level in osteoarthritic cartilage has been described by earlier workers. ${ }^{2}$ We showed the presence of crystals, probably apatite, in macroscopically normal articular cartilage of the aged human hip joint.

Femoral heads were obtained from eight women undergoing operation for hip arthroplasty after subcapital fracture. Ages ranged from 65 to 91 years and in four cases fracture of the femoral neck had occurred the day before operation. The cartilage of the whole femoral head appeared smooth, though in several specimens a small area about 3-4 $\mathrm{mm}$ diameter beneath the fovea showed minimal fibrillation. Tissue blocks of smooth cartilage from the superior and inferior surface of the femoral head were fixed in glutaraldehyde, stained with osmic acid, and embedded in araldite. Thin sections were stained with uranyl ace tate and lead citrate and examined by transmission electron microscopy. Unstained sections of non-osmicated tissue fixed in unbuffered $10 \%$ formaline were also examined.
Crystals were found in all specimens, occurring singly or occasionally in clusters. They were mostly $0.2 \mu \mathrm{m}$ in diameter but varied from about 0.05 to $15 \mu \mathrm{m}$. They were often polygonal or rounded in shape but many had a sharply angled square contour and were considered to be cuboidal. Several crystals were enclosed by an electron dense lamina, possibly a membrane, adherent to the surrounding cartilage matrix. Many 'empty' areas lined by a similar dense lamina were observed; these corresponded in size and shape to the crystals. This may have been due to 